\title{
Galactic magnetic field generation by the electric current of cosmic rays
}

\author{
A. Z. Dolginov ${ }^{1}$ and I. N. Toptygin ${ }^{2}$ \\ 1 125, B-2, Hillcrest Vil.E., Schenectady, NY, 12309, USA \\ 2 St. Petersburg State Polytechnical University 195251, St. Petersburg, Russia
}

Received 4 March 2003 / Accepted 20 November 2003

\begin{abstract}
In this paper we propose a new mechanism for the generation of a large-scale galactic magnetic field by the electric current created by cosmic rays. A model with the sources of relativistic particles, distributed homogenously and continuously in the galactic disk is used, and intensities of the sources correspond to the observed intensities. The anisotropic diffusion of cosmic rays in the galaxy is considered and it is shown that the created magnetic field depends strongly on the symmetry of the diffusion tensor as well as on the relationship between the tensor's components. The helicity of turbulence ( $\alpha$-effect) is not considered. Only the small-scale "seed" fields ejected from stars and generated by cosmic rays are necessary to start the global magnetic field generation. The diffusion of the field because of turbulent motions and the differential rotation of the galactic disk are taken into account. The diffusion coefficient values estimated from the observed parameters of the galactic medium are used in the calculations. The obtained magnetic field is approximately a few micro-gauss which is of the same order of magnitude as the observed galactic field. Therefore, it is concluded that the relativistic particles are significant and, possibly, essential for the process of the large-scale galactic magnetic field generation. More precise determinations of the diffusion coefficients for the field and the particles are needed to reach the final conclusion about the field's value. The future development of the theory should also include dynamo action, the nonlinear effects, and the self-consistent approach to the problem.
\end{abstract}

Key words. ISM: cosmic rays - diffusion - Galaxy: kinematics and dynamics - ISM: magnetic field

\section{Introduction}

Observations show that galaxies have both irregular small-scale and regular large-scale magnetic fields, as discussed, for example, in the book of Rusmaikin et al. (1988), in reviews of Beck et al. (1996); and in Beck (2001). These observations are usually explained by the dynamo theory (see Beck et al. 1996). A review of theoretical methods is contained in Parker's (1979) book. The observed regular field has a predominantly toroidal structure. In some spiral galaxies this field has a symmetrical structure, while in others it is bisymmetrical. The differential rotation of the galactic disk can transform the poloidal component of the field to a toroidal one. The data on the poloidal field is not very accurate since it is difficult to observe the field component which is perpendicular to the galactic plane. The data on the plasma turbulent velocities in the galaxy, which determine the dynamo action, is also not very precise. Some reasonable assumptions (Rusmaikin et al. 1988; Beck et al. 1996) were used to compensate for the incompleteness of observational data, however, these assumptions make it impossible for the theory to be fully confirmed by observational data. The existing theory meets some other challenges. The nonlinear effects may stop the large-scale field generation by the dynamo process (Grusinov \& Diamond 1994, 1996). The small-scale dynamo field increases much faster than the large-scale one and this small-scale field may kill the turbulence that is necessary to create the large-scale field (see: Vainstein \& Rossener 1991; Vainstein \& Cattaneo 1992; Kulsrud \& Anderson 1992). A large-scale regular field was observed in the Magellan Clouds (Chi \& Wolfendale 1993), which are irregular galaxies; however, no noticeable differential rotation needed for dynamo action was observed there.

The difficulties of the existing theories led us to consider field generation processes which are different from a dynamo. In this paper it will be shown that the electric current created by cosmic rays can generate a significant large-scale magnetic field.

The power, composition, and energy spectrum of cosmic rays have been thoroughly investigated by many authors. Most of the relativistic particles in the galaxy are protons, which have an energy value on the order of $1 \mathrm{GeV}$. The acceleration process is located mainly in the galactic disk (e.g. in supernova remnants, strong stellar winds, etc.). The cosmic rays diffuse enormous distances to the galaxy boundaries and leave the stellar system in $(1-3) \times 10^{8}$ years. The total energy power of relativistic particle

Send offprint requests to: A. Z. Dolginov, e-mail: adolgi1@yahoo.com 
production in the galaxy is estimated to be $Q_{\varepsilon} \approx(1-3) \times 10^{40} \mathrm{erg} \mathrm{s}^{-1}$ (Berezinsky et al. 1990). The total power of relativistic particle production is on the order of $Q_{0} \approx 10^{43}$ particles per second.

Energetic particles spread over the galaxy and interact with the background plasma. They create an effective electric current, which can act as a source of a large-scale magnetic field. The value of the galactic magnetic field will be calculated and compared with observational data.

While the resonance generation of plasma waves and small-scale magnetic fields of the scale of the Larmor radius of the relativistic particles has been thoroughly investigated by many authors (see, for example, Völk \& McKenzie 1982), large-scale field generation in the process of cosmic rays propagation has never been considered. We will consider the contribution of cosmic rays separately from other possible effects, such as dynamo action, which may also be important for the generation of the galactic magnetic field. In a more general theory all these effects would have to be considered.

\section{The accepted model and basic equations}

The interstellar medium contains two kinds of plasma: the thermal electron-ion plasma and the plasma of cosmic rays. The hydrodynamical pressure of the cosmic rays is in equipartition with the pressure of the interstellar thermal plasma. Protons and helium ions are the main component of the relativistic particles in the galaxy (Berezinskii et al. 1990). The number density of helium ions and other heavy ions is much less than that of the protons. The contribution of relativistic electrons is no more than $1 \%$ of the total number of relativistic particles. The number of relativistic particles decreases with the energy $E$ as $E^{-2.7}$. This allows the use of a simple model of the galactic plasma consisting of relativistic protons, with average energy $E=1 \mathrm{GeV}$, and number density $N=10^{-9} \mathrm{~cm}^{-3}$, and particles of interstellar thermal plasma with proton and electron number densities $n \gg N$. The real distribution of the discrete cosmic ray sources in the galaxy is not well known. A stationary model with parameters averaged over the volume of the galactic disk will be used. The disk is described by its half-thickness $h$ in the $z$ direction and by the effective radius $R$. The distribution of the cosmic-ray sources in the disk is assumed to have azimuthal symmetry. Such distribution in the cylindrical coordinate system is in the form

$Q(r, z)=\frac{Q_{0}}{8}\left(\frac{R}{h}\right) \frac{\Theta(h-|z|) \cos (\pi z / 2 h)}{\left(r^{2}+R^{2}\right)^{3 / 2}}$,

where $\Theta(x)=1$ if $x>0$ and $\Theta(x)=0$ if $x<0$. The cosmic ray sources are located inside the disk of thickness $2 h$ and are absent outside the disk. The density of the sources decreases for $r \gg R$ as $r^{-3}$. The real density decrease is apparently sharper but the disk periphery is not important for this problem. The source of non-relativistic protons $Q_{\mathrm{p}}^{\prime}$ has to be equal to the source of relativistic protons with the opposite sign:

$Q_{\mathrm{p}}(r, z)=-Q_{\mathrm{p}}^{\prime}(r, z)=Q(r, z)$.

The Fourier image of the expression (1) is

$Q_{k}=\frac{\pi Q_{0}}{2 h} \mathrm{e}^{-k_{\perp} R} \int_{0}^{h} \cos \left(\frac{\pi \zeta}{2 h}\right) \cos k_{\|} \zeta \mathrm{d} \zeta$

Here $\left(k_{\perp}, \phi, k_{\|}\right)$are cylindrical components of the wave vector $\boldsymbol{k}$, where $k_{\|}$is the $\boldsymbol{k}$ component parallel and $k_{\perp}$ is perpendicular to the plane of the disk.

The magnetic field is frozen in the plasma component whose conductivity is larger. In particular, the magnetic field in the galaxy is frozen in the thermal plasma. Thermal electrons and ions cannot diffuse separately (this effect is known as ambipolar diffusion) and the average current is zero if there are no sources of particles in the plasma. The cosmic rays have sources in the galaxy and they leave the galaxy after some time of travelling.

Relativistic and thermal particles move in the galaxy by anisotropic diffusion. The diffusion coefficients $\kappa_{\mu v}$ of relativistic protons and $D_{\mu \nu}^{\mathrm{p}, \mathrm{e}}$ of thermal protons and electrons are dependent on the regular magnetic field, the small-scale random magnetic field, the turbulent motion of background plasma, and on the collisions with neutral and charged particles. The regular motions, such as galactic wind, are not included in the model, however, the differential rotation of the disk is taken into account.

The diffusion rate of electrons and thermal protons is smaller than that of cosmic ray ions. This difference leads to the separation of charged particles and, hence, to the creation of the electric field. In this paper it will be shown that an average electric current generated by cosmic ray protons in the galaxy is not compensated for by the electrons and that this current can create a significant large-scale magnetic field. The energy losses of cosmic ray ions with energy above $100 \mathrm{MeV}$ can be ignored. Cosmic ray protons leave the galaxy easily. Magnetic inhomogeneities with sizes on the order of the Larmor radius of the particle are the most effective for scattering. This radius is large for cosmic ray particles. Unfortunately, the small-scale magnetic field distribution is not well known. The different scales of magnetic fluctuations are responsible for scattering electrons and ions and are an additional cause for the differences in diffusion of ions and electrons. Proton and electron currents can be presented in the form

$j_{\mu}^{\mathrm{p}}=-e \kappa_{\mu \nu} \frac{\partial N}{\partial x_{\nu}}-e D_{\mu \nu}^{\mathrm{p}} \frac{\partial n^{\mathrm{p}}}{\partial x_{v}}+\sigma_{\mu \nu}^{\mathrm{p}} E_{v}$, 
$j_{\mu}^{\mathrm{e}}=e D_{\mu \nu}^{\mathrm{e}} \frac{\partial n^{\mathrm{e}}}{\partial x_{v}}+\sigma_{\mu \nu}^{\mathrm{e}} E_{\nu}$

Only a small fraction of the total number of plasma protons takes part in the acceleration process. The increase of the cosmic ray protons is due to the decrease of the thermal protons. The change of the proton number density creates an electric field which interacts with all the protons and electrons. $n^{\mathrm{p}, \mathrm{e}}$ in the formulas (4) and (5) are the number densities of the perturbed parts of the thermal particles. $\sigma_{\mu \nu}^{\mathrm{p}, \mathrm{e}}$ are the coefficients of conductivity for the proton and electron currents in the interstellar plasma, $E_{v}$ is the electric field component. Macroscopic electric fields satisfy the electrostatic equations

$\boldsymbol{E}=-\nabla \varphi, \quad \nabla^{2} \varphi=-4 \pi e\left(N+n^{\mathrm{p}}-n^{\mathrm{e}}\right)$.

The total current $\boldsymbol{j}^{\mathrm{cr}}=\boldsymbol{j}^{\mathrm{p}}+\boldsymbol{j}^{\mathrm{e}}$ is divergence-free. $\nabla \cdot \boldsymbol{j}^{\mathrm{cr}}=0$. Both proton and electron currents are also divergent free, but the curl of the currents is not zero.

$\nabla \cdot \boldsymbol{j}^{\mathrm{p}}=0, \quad \nabla \cdot \boldsymbol{j}^{\mathrm{e}}=0$.

As discussed above, the diffusion coefficients depend on the large-scale and small-scale magnetic fields, which depend, in turn, on the ambient plasma density. The plasma density decreases to the periphery of the disk. The thickness of the disk decreases much faster in the $z$ direction than in the radial and azimuthal directions, which leads to different free paths in different directions and, therefore, to a difference of the diffusion coefficients. We use a simplified model because the disk is very inhomogeneous and observational data about the small-scale fields is not well known. However, the difference of the diffusion coefficients, which are dependent on the geometry of the disk, has to be taken into account. The diffusion coefficients are assumed to be tensors. The tensor components have different values, but each component is considered to be constant averaged over the corresponding direction in space. Under these assumptions we obtain

$-e \kappa_{\mu \nu} \frac{\partial^{2} N}{\partial x_{\mu} \partial x_{v}}=e Q$,

$-e D_{\mu \nu}^{\mathrm{p}} \frac{\partial^{2} n^{\mathrm{p}}}{\partial x_{\mu} \partial x_{v}}-\sigma_{\mu \nu}^{\mathrm{p}} \frac{\partial^{2} \varphi}{\partial x_{\mu} \partial x_{v}}=-e Q$,

$e D_{\mu \nu}^{\mathrm{e}} \frac{\partial^{2} n^{\mathrm{e}}}{\partial x_{\mu} \partial x_{v}}-\sigma_{\mu \nu}^{\mathrm{e}} \frac{\partial^{2} \varphi}{\partial x_{\mu} \partial x_{v}}=0$.

The electric field $\boldsymbol{E}$ can be disregarded for the process of the relativistic proton propagation (see Berezinsky et al. 1990, and Sect. 3 of this paper).

Obtaining the $N, n^{\mathrm{p}}, n^{\mathrm{e}}$ and $\varphi$ values from Eqs. (4) through (10), the magnetic field created by the total macroscopic current $j^{\mathrm{cr}}$ in the turbulent galactic medium can be calculated. The turbulence plays a significant role in the problem because it determines the dissipation of the field and the diffusion of the particles.

The local magnetic field (not averaged over the turbulent motion) satisfies the equation

$\nabla \times \boldsymbol{H}=\frac{4 \pi}{c}\left(\boldsymbol{j}^{\mathrm{in}}+\boldsymbol{j}^{\mathrm{ext}}\right)$,

where

$j_{\mu}^{\text {in }}=\tilde{\sigma}_{\mu \nu}\left(\boldsymbol{E}+\frac{1}{c} \boldsymbol{v} \times \boldsymbol{H}\right)_{v}$

is the local current that exists in the plasma even in the absence of relativistic particles, and $\boldsymbol{j}^{\text {ext }}$ is the additional current due to the presence of cosmic rays. The quantity $\tilde{\sigma}_{\mu \nu}$ in Eqs. (10)-(12) is the local coefficient of conductivity, which is different in the different interstellar regions. Averaging Eq. (11) over the turbulent motions of the medium yields

$\nabla \times \boldsymbol{B}=\frac{4 \pi}{c}\left(\boldsymbol{j}^{\mathrm{pl}}+\boldsymbol{j}^{\mathrm{cr}}\right)$,

where $\boldsymbol{B}=\langle\boldsymbol{H}\rangle, \boldsymbol{j}^{\mathrm{pl}}=\left\langle\boldsymbol{j}^{\text {in }}\right\rangle, \boldsymbol{j}^{\text {cr }}=\left\langle\boldsymbol{j}^{\text {ext }}\right\rangle$ are averaged values. Throughout the paper, angular brackets are used to denote the operation of averaging. The current $\boldsymbol{j}^{\mathrm{cr}}$ has to be calculated from Eqs. (4)-(10), and the current $\boldsymbol{j}^{\mathrm{pl}}$ can be calculated using the average of the expression

$\mathcal{E}=\hat{\sigma}^{-1} \boldsymbol{j}^{\text {in }}-\frac{1}{c} \boldsymbol{v} \times \boldsymbol{H}$ 
We assume that $\sigma_{\mu \nu}=$ const. (it corresponds to constant density and temperature), $\boldsymbol{v}=\boldsymbol{u}+\boldsymbol{u}^{\prime}$, where $\boldsymbol{u}=\langle\boldsymbol{v}\rangle$ is the regular and $\boldsymbol{u}^{\prime}$ is the turbulent component of the plasma velocity, $\left\langle\boldsymbol{u}^{\prime}\right\rangle=0$. The expression for the average value $\left\langle\boldsymbol{u}^{\prime} \times \boldsymbol{H}\right\rangle$ can be found, for example, in books of Vainstein et al. (1980); Rusmaikin et al. (1988) and many others. In the case of isotropic turbulence

$\left\langle\boldsymbol{u}^{\prime} \times \boldsymbol{H}\right\rangle=-v_{\text {turb }} \nabla \times \boldsymbol{B}, \quad v_{\text {turb }}=\frac{1}{3}\left\langle u^{\prime 2}\right\rangle \tau_{\mathrm{c}}$.

Here $\tau_{\mathrm{c}}$ is the correlation time of the turbulent motions, $v_{\mathrm{turb}}$ is the coefficient of the magnetic field's turbulent diffusion (turbulent magnetic viscosity). Averaging Eq. (14) with the help of Eqs. (15) and (11) we obtain

$c\langle\boldsymbol{E}\rangle=c \boldsymbol{E}=-c\left\langle\widetilde{\sigma}^{-1} \boldsymbol{j}^{\mathrm{ext}}\right\rangle+\frac{c^{2}}{4 \pi}\left\langle\widetilde{\sigma}^{-1} \nabla \times \boldsymbol{H}\right\rangle-\boldsymbol{u} \times \boldsymbol{B}+v_{\mathrm{turb}} \nabla \times \boldsymbol{B}$

The conductivity depends on the plasma density and on the magnetic fields, but does not directly depend on the current.

To estimate the term being averaged, we use the scalar $\widehat{\sigma}$ instead of the tensor $\sigma_{\mu \nu}$ and disregard the correlations of $\sigma_{\mu \nu}$ with $\boldsymbol{j}^{\text {ext }}$ and with $\nabla \times \boldsymbol{H}$. In this case

$\left\langle\widetilde{\sigma}^{-1} \boldsymbol{j}^{\mathrm{ext}}\right\rangle=\frac{1}{\sigma} \boldsymbol{j}^{\mathrm{cr}}, \quad\left\langle\widetilde{\sigma}^{-1} \nabla \times \boldsymbol{H}\right\rangle=\frac{1}{\sigma} \nabla \times \boldsymbol{B}$

Applying the operation $\nabla \times$ to Eq. (16) and using the Faraday law for $\nabla \times \boldsymbol{E}$, we obtain

$\frac{\partial \boldsymbol{B}}{\partial t}=v \nabla^{2} \boldsymbol{B}+\nabla \times[\boldsymbol{u} \times \boldsymbol{B}]+\frac{4 \pi v_{\mathrm{m}}}{c} \nabla \times \boldsymbol{j}^{\mathrm{cr}}$.

Here $v_{\mathrm{m}}=c^{2} / 4 \pi \sigma$ is the local magnetic viscosity, and $v=v_{\mathrm{m}}+v_{\mathrm{turb}}$ is the complete magnetic viscosity. The local magnetic viscosity is many orders of magnitude less than the turbulent viscosity $v_{\text {turb }}$ in the galaxy, and, therefore, $v \approx v_{\text {turb }}$.

In the absence of regular motions and in the static case $(\partial \boldsymbol{B} / \partial t=0)$ we obtain

$\nabla^{2} \boldsymbol{B}=-\frac{4 \pi}{c} \frac{v_{\mathrm{m}}}{v_{\text {turb }}} \nabla \times \boldsymbol{j}^{\mathrm{cr}}$

The small factor $v_{\mathrm{m}} / v_{\text {turb }}$ in Eq. (19) is connected with the large-scale field dissipation due to turbulent diffusion.

\section{The electric current density}

Equations (6)-(10) are solved under the assumption that the galactic disk is homogeneous, i.e., that the components of the tensors of diffusion and conductivity are independent of the coordinates inside the disk. The simplest way to get the solution of Eqs. (6)(10) is the method of Fourier transformation. Using the three-dimensional integral Fourier expansion and Eq. (10) we obtain $-D_{\mu \nu}^{\mathrm{e}} k_{\mu} k_{\nu} n_{k}^{\mathrm{e}}+\sigma_{\mu \nu}^{\mathrm{e}} k_{\mu} k_{\nu} \varphi_{k}=0$, i.e.

$\varphi_{\boldsymbol{k}}=\frac{D_{\mu \nu}^{\mathrm{e}} k_{\mu} k_{v}}{\sigma_{\alpha \beta}^{\mathrm{e}} k_{\alpha} k_{\beta}} n_{k}^{\mathrm{e}}$.

We can use the relation (Alfen \& Falthammar 1963):

$\sigma_{\mu \nu}^{\mathrm{e}, \mathrm{p}}=\frac{D_{\mu \nu}^{\mathrm{e}, \mathrm{p}}}{4 \pi r_{\mathrm{D}}^{2}}$.

Here $r_{\mathrm{D}}=\sqrt{T / 4 \pi n_{0} e^{2}}$ is the Debye radius, $n_{0}$ is the equilibrium electron and ion number density, $T$ is the temperature in the units of energy. The formula (21) is a direct consequence of the well known Einstein relation of the particle's mobility and diffusivity. From Eqs. (20), (21), and (5) we obtain $\varphi_{\boldsymbol{k}}=4 \pi r_{\mathrm{D}}^{2} n_{\boldsymbol{k}}^{\mathrm{e}}$ and

$j_{k \mu}^{\mathrm{e}}=i e D_{\mu \nu}^{\mathrm{e}} k_{v} n_{k}^{\mathrm{e}}-i \sigma_{\mu \nu}^{\mathrm{e}} k_{\nu} \varphi_{k}=0$

The absence of electron sources, the relation (21) of the kinetic coefficients, and the assumption that the kinetic coefficients are constant inside the disk of the galaxy lead to the zero electron current. Only a very small fraction of the total number of plasma protons takes part in the acceleration process. The increase of the cosmic ray protons is equal to the decrease of thermal protons. The change of the proton number density, due to acceleration, creates an electric field, which interacts with plasma protons and electrons. The essential difference between the perturbed plasma electrons and protons is the difference in the number densities. The number density of accelerated electrons is only one percent of the number density of accelerated protons. We can disregard 
the change of the plasma electron number density, but take into account the influence of the electric field. The contribution of the electron current due to electric fields is compensated by its contribution due to diffusion. The proton current remains. In this case

$$
\begin{aligned}
N_{k} & =\frac{Q_{k}}{\widetilde{\kappa}}, \\
n_{k}^{\mathrm{p}} & =-\frac{Q_{k}}{2+k^{2} r_{\mathrm{D}}^{2}}\left(\frac{1}{\widetilde{\kappa}}+\frac{1+k^{2} r_{\mathrm{D}}^{2}}{\widetilde{D}_{\mathrm{p}}}\right), \\
n_{k}^{\mathrm{e}} & =\frac{Q_{k}}{2+k^{2} r_{\mathrm{D}}^{2}}\left(\frac{1}{\widetilde{\kappa}}-\frac{1}{\widetilde{D}_{\mathrm{p}}}\right), \\
\varphi_{k} & =\frac{4 \pi e}{k^{2}}\left(N_{k}+n_{k}^{\mathrm{p}}-n_{k}^{\mathrm{e}}\right)=\frac{4 \pi e Q_{k}\left(k r_{\mathrm{D}}\right)^{2}}{k^{2}\left(2+k^{2} r_{\mathrm{D}}^{2}\right)}\left(\frac{1}{\widetilde{\kappa}}-\frac{1}{\widetilde{D}_{\mathrm{p}}}\right) .
\end{aligned}
$$

Here

$\widetilde{\kappa}=\kappa_{\mu v} k_{\mu} k_{v}, \quad \widetilde{D}_{\mathrm{p}}=D_{\mu \nu}^{\mathrm{p}} k_{\mu} k_{v}$.

Summation over repeated indices is used throughout. The diffusion coefficient for relativistic protons in the galaxy is much larger than for thermal protons, i.e. $\widetilde{K} \gg \widetilde{D}_{\mathrm{p}}$. Thus, the number density of the non-thermal parts $n_{k}^{\mathrm{p}}$ and $n_{k}^{\mathrm{e}}$ of the interstellar plasma particles are large compared to the number density of relativistic protons $N_{k}$. According to Eq. (26), a self-consistent field has a small factor $\left(k r_{\mathrm{D}}\right)^{2}=\left(2 \pi r_{\mathrm{D}} / L\right)^{2} \approx 10^{-34}$, if $r_{\mathrm{D}} \approx 10^{3} \mathrm{~cm}$ (warm phase), and $L \approx 0.2 \mathrm{kpc}$ (half-thickness of the disk). The relative effect of the electric field on relativistic protons is determined by the factor $e E L / m_{\mathrm{p}} c^{2} \approx 16 \pi e^{2} N r_{\mathrm{D}}^{2} / m_{\mathrm{p}} c^{2} \approx 10^{-16}$, where $N \approx 10^{-9} \mathrm{~cm}^{-3}$ is the cosmic ray proton number density. We consider the effect of the electric field in Eq. (8) for relativistic protons to be negligible. Using expressions (23)-(27) and formulas (4) and (5), we can obtain the Fourier transform of the total current

$j_{\boldsymbol{k} \mu}^{\mathrm{cr}}=j_{\boldsymbol{k} \mu}^{\mathrm{e}}+j_{\boldsymbol{k} \mu}^{\mathrm{p}}=i e Q_{k}\left(\frac{D_{\mu \nu}^{\mathrm{p}} k_{v}}{\widetilde{D}_{\mathrm{p}}}-\frac{\kappa_{\mu \nu} k_{v}}{\widetilde{\kappa}}\right)$.

It follows from Eq. (28) that the electric current is created by relativistic and non-relativistic protons and electrons of the interstellar plasma. According to Eqs. (18) and (19), the magnetic field in our model is determined by the curl of the current. Note the following important property of the derived current: its curl becomes zero if the medium is homogeneous and the tensors of diffusion and conductivity are diagonal. Calculating $\left[\boldsymbol{k} \times \boldsymbol{j}_{\boldsymbol{k}}^{\mathrm{cr}}\right]_{\alpha}=e_{\alpha \beta \mu} k_{\beta} j_{k \mu}^{\mathrm{cr}}$, we obtain the zero combination of the tensors $e_{\alpha \beta \mu} k_{\beta} k_{\mu}=0$ for $\kappa_{\mu \nu}=\kappa \delta_{\mu v}$.

Thus, a magnetic field in a homogeneous system can be generated by a current created in the diffusion process of energetic particles only if the medium has anisotropic properties. This assertion is valid irrespective of the distribution of the sources. This result can be easily understood from qualitative considerations. In the case of isotropic diffusion, any small source of relativistic particles will produce a spherically symmetrical current that does not generate any magnetic field. Therefore, the field strength is very sensitive to the symmetry of the diffusion tensors and to the relationship between their components.

It should be noted, however, that in an inhomogeneous system, which the galaxy and, in particular, the galactic disk actually are, the curl of the current can be nonzero if the diffusion coefficients depend on the coordinates. In this case instead of Eq. (4) we have

$\boldsymbol{j}^{\mathrm{p}}=-e \kappa \nabla N-e D^{\mathrm{p}} \nabla n^{\mathrm{p}}+\sigma^{\mathrm{p}} \boldsymbol{E}$.

The curl of the current density is not zero

$\nabla \times \boldsymbol{j}^{\mathrm{p}}=-e \nabla \kappa \times \nabla N-e \nabla D^{\mathrm{p}} \times \nabla n^{\mathrm{p}}+\nabla \sigma^{\mathrm{p}} \times \boldsymbol{E} \neq 0$,

if the gradients of the diffusion coefficients and the gradient of the particle number density are not parallel. The characteristic scales of the particle number densities $N, n^{\mathrm{p}}$ and the diffusion coefficients are determined by the size and geometry of the disk. All scales are approximately of the same order of magnitude. Therefore, the curls of Eqs. (28) and (30) are also approximately of the same order of magnitude. This means that the magnetic fields may be generated in an isotropic, but nonuniform galactic medium. Such a possibility is of great importance especially during the initial periods of the existence of the disk. In such a medium, an asymmetric electric current of accelerated particles could be the source of the large-scale magnetic field. The small-scale field appears to be ejected from stars.

In this paper only the effect of anisotropic propagation of cosmic rays in a homogeneous medium is considered. The calculations of electric current and magnetic field in the inhomogeneous medium is a separate and more complicated problem, which is out of the scope of this paper. 


\section{Diffusion coefficients}

The relativistic protons have the longest free path in the galaxy. In this case the efficient diffusion coefficient $\kappa \approx 5 \times 10^{28} \mathrm{~cm}^{2} \mathrm{~s}^{-1}$ (Beresinsky et al. 1990). The local tensor of diffusion in the galaxy is strongly anisotropic: $\kappa_{\perp} / \kappa_{\|} \approx 10^{-13}$, where the indices $\perp$ and $\|$ determine the directions perpendicular and parallel to the large-scale field respectively. Magnetic field fluctuations are of the same order of magnitude as the average field, $\delta B / B_{0} \approx 1$. It makes the average diffusion tensor much more isotropic. In this case $\kappa_{\perp}$ is of the same order of magnitude as $\kappa_{\|}$, but not equal to it. This problem was considered in detail by Bykov \& Toptygin (1992) and equations for $\kappa_{\perp}$ and $\kappa_{\|}$were obtained. These authors used a renormalization method to calculate the average diffusion tensor for strong turbulence. This method leads to a system of transcendental equations for the components of the diffusion tensor:

$$
\begin{aligned}
\kappa_{\alpha \beta}^{\perp}= & \kappa_{\perp}^{0} \delta_{\alpha \beta}^{\perp}+\left(1-\frac{\epsilon}{2}\right)^{2} \int \frac{\left\langle u_{\alpha}^{\prime} u_{\beta}^{\prime}\right\rangle_{k \omega}}{\mathrm{i} \omega+k_{\|}^{2} \kappa_{\|}+k_{\perp}^{2} \kappa_{\perp}} \frac{\mathrm{d}^{3} k \mathrm{~d} \omega}{(2 \pi)^{4}} \\
& +\kappa_{\|}^{0} \frac{1-\epsilon-\left\langle B^{2} \delta B^{2} /\left(B^{2}+\delta B^{2}\right)^{2}\right\rangle}{(1-\epsilon)^{2}} \int \frac{\left\langle\delta B_{\alpha} \delta B_{\beta}\right\rangle_{k \omega}}{B^{2}}\left(1-\frac{k_{\|}^{2} \kappa_{\|}^{0}}{\mathrm{i} \omega+k_{\|}^{2} \kappa_{\|}+k_{\perp}^{2} \kappa_{\perp}}\right) \frac{\mathrm{d}^{3} k \mathrm{~d} \omega}{(2 \pi)^{4}},
\end{aligned}
$$

where

$$
\begin{aligned}
& \kappa_{\|}=\kappa_{\|}^{0}\left[1+\frac{\left\langle\delta B^{2}\right\rangle}{B^{2}} \frac{1-\epsilon-\left\langle B^{2} \delta B^{2} /\left(B^{2}+\delta B^{2}\right)^{2}\right\rangle}{(1-\epsilon)^{2}}\right] \\
& \epsilon=\left\langle\delta B^{2} /\left(B^{2}+\delta B^{2}\right)\right\rangle, \kappa_{\|}^{0}=\frac{v^{2}}{3 v_{0}}(1-\epsilon)^{2}, \kappa_{\perp}^{0}=\frac{v^{2}}{3 v_{0}}\left(\frac{\epsilon}{2}\right)^{2} .
\end{aligned}
$$

Here $v_{0}$ is the frequency of relativistic particles scattering by small-scale fields, $\delta \boldsymbol{B}$ is the stochastic large-scale magnetic field, transversal to the regular uniform field $\boldsymbol{B}$, and $\boldsymbol{u}^{\prime}$ is the turbulent velocity. Equation (31) includes correlation tensors of the magnetic field and correlation tensors of the turbulent velocity. Equation (31) determines the transversal component $\kappa_{\perp}$ of the diffusion tensor and is valid over the entire range $0 \leq \epsilon \leq 1$, i.e. without any restriction on the amplitude of the magnetic field or turbulent velocity.

The turbulent transfer is insignificant for relativistic protons in galaxy. We assume $u^{\prime}=0$ and consider a static random velocity field with a single correlation scale:

$\left\langle\delta B_{\alpha} \delta B_{\beta}\right\rangle_{k \omega}=\left\langle\delta B^{2}\right\rangle \frac{2 \pi^{3}}{k_{0}^{2}} \delta\left(k-k_{0}\right) \delta(\omega) \delta_{\alpha \beta}^{\perp}$.

Here the correlation length $L_{0}=2 \pi / k_{0}$ significantly exceeds the local free path $\Lambda_{\|}=v / v_{0}$. The correlation tensor (34) is normalized by the condition

$\int\left\langle\delta B_{\alpha} \delta B_{\alpha}\right\rangle_{k \omega} \frac{\mathrm{d}^{3} k \mathrm{~d} \omega}{(2 \pi)^{4}}=\left\langle\delta B^{2}\right\rangle$.

After substituting Eq. (34) in Eq. (31) and integrating, we obtain the transcendental equation for the ratio of the diffusion coefficients $X=\kappa_{\perp} / \kappa_{\|} \leq 1$ :

$X=\frac{\epsilon^{2}}{4(1-\epsilon)}+\frac{\epsilon}{2}\left\{\frac{\epsilon-X}{1-X}+\frac{(1-\epsilon) \sqrt{X}}{(1-X)^{3 / 2}} \arctan \sqrt{\frac{1-X}{X}}\right\}$

We consider the case of strong turbulence in the galaxy and take from observations (Ruzmaikin et al. 1988; Beck et al. 1996; Beck 2001) values of regular and random magnetic fields $\delta B \approx B(\epsilon \approx 1 / 2) ; \delta B \approx 1.4 B(\epsilon \approx 2 / 3) ; \delta B \approx 1.7 B(\epsilon \approx 3 / 4)$. We obtain from Eq. (31) values $\kappa_{\perp} / \kappa_{\|} \approx 0.3, \kappa_{\perp} / \kappa_{\|} \approx 0.6, \kappa_{\perp} / \kappa_{\|} \approx 0.9$ respectively. Thus, $\kappa_{\perp}$ and $\kappa_{\|}$are of the same order of magnitude. The tensor components for the Hall diffusion are negligible, because the Larmor radius of relativistic protons is too small compared with the mean free path of the protons. The average diffusion tensor of the relativistic protons is diagonal in the coordinate system with the polar axis directed along the large-scale regular field.

The observed galactic large-scale magnetic field has spiral field lines in the plane of the disk (Rusmaikin et al. 1988). The data about the field perpendicular to the disk are not well known because this component is much weaker than the field in the plane of the disk. We will consider all six components of the symmetric tensors $D_{\mu \nu}^{\mathrm{e}}$ and $\kappa_{\mu \nu}$, including the cross components such as $\left(\kappa_{r \alpha}, \kappa_{r z}, \kappa_{\alpha z}\right)$. These components are very important. It follows from the well known anti-dynamo theorems (Elsasser 1946; Zeldovich 1956) that the asymmetry of the system is necessary for the magnetic field generation. In our case the symmetry of the diffusion tensor has to be different from the cylindrical symmetry of the accepted model of the galaxy. The components of the diffusion tensor can be presented in the form

$$
\kappa_{\mu v}=\left(\begin{array}{lcr}
\left(\kappa_{\|}-\kappa_{\perp}\right) \sin ^{2} \theta \cos ^{2} \gamma+\kappa_{\perp} & \left(\kappa_{\|}-\kappa_{\perp}\right) \sin ^{2} \theta \sin \gamma \cos \gamma & \left(\kappa_{\|}-\kappa_{\perp}\right) \sin \theta \cos \theta \cos \gamma \\
\left(\kappa_{\|}-\kappa_{\perp}\right) \sin ^{2} \theta \sin \gamma \cos \gamma & \left(\kappa_{\|}-\kappa_{\perp}\right) \sin ^{2} \theta \sin ^{2} \gamma+\kappa_{\perp} & \left(\kappa_{\|} \sin \gamma-\kappa_{\perp} \cos \gamma\right) \sin \theta \cos \theta \\
\left(\kappa_{\|}-\kappa_{\perp}\right) \sin \theta \cos \theta \cos \gamma & \left(\kappa_{\|} \sin \gamma-\kappa_{\perp} \cos \gamma\right) \sin \theta \cos \theta & \kappa_{\|} \cos ^{2} \theta+\kappa_{\perp} \sin ^{2} \theta
\end{array}\right) .
$$


Here $\theta$ is the angle between the line of force and the $z$ direction, and $\gamma$ is the angle between the field projection on the plane $(x, y)$ and the $\boldsymbol{r}$ direction in the cylindrical coordinate system $(r, \alpha, z)$. If the toroidal component $B_{\alpha}$ is predominant, then the angles $\theta$ and $\gamma$ are close to $\pi / 2$. The structure of the tensor $D_{\mu \nu}^{\mathrm{e}}$ for nonrelativistic electrons is similar to that of $\kappa_{\mu \nu}$ because it is also determined by the large-scale field structure. Components of $D_{\mu \nu}^{\mathrm{e}}$ are much smaller than $\kappa_{\mu \nu}$ but this is not relevant because the electric current, according to Eq. (28), depends only on the ratio of the tensor components.

The magnetic field value also depends on the viscosities $v_{\mathrm{m}}$ and $v_{\text {turb }}$. The $v_{\text {turb }}$ can be estimated using the formula $v_{\text {turb }} \approx$ $u^{\prime} L / 3$, where $L \approx 100 \mathrm{pc}$ for the most important component of the interstellar turbulence and $u^{\prime} \approx 10^{6} \mathrm{~cm} \mathrm{~s}^{-1}$. It gives $v_{\text {turb }} \approx 10^{26}$ $\mathrm{cm}^{2} \mathrm{~s}^{-1}$. The viscosity $v_{\mathrm{m}}$ is different in the different regions of the galaxy. There are clouds of neutral hydrogen, warm areas with ionized hydrogen $\left(n_{\mathrm{e}} \approx 0.02 \mathrm{~cm}^{-3}\right)$, and hot regions $\left(T \approx 10^{6} \mathrm{~K}, n_{\mathrm{e}} \approx 0.002 \mathrm{~cm}^{-3}\right)$ created by supernova remnants. Hot regions occupy a significant part of the disk's volume. The magnetic field dissipation is predominantly due to the ambipolar diffusion of electrons and ions through the neutral gas in the "cold" dense non-ionized hydrogen clouds and in the "warm" regions where the percentage of the non-ionized atoms is high. The ambipolar diffusion in these regions is determined by: $v_{\mathrm{m}} \approx 3 \times 10^{21} \mathrm{~cm}^{2} \mathrm{~s}^{-1}$ and $v_{\mathrm{m}} \approx 3 \times 10^{20} \mathrm{~cm}^{2} \mathrm{~s}^{-1}$ respectively (Rusmaikin et al. 1988). The collisionless damping of plasma waves in the hot regions gives $v_{\mathrm{m}} \approx 5 \times 10^{17} \mathrm{~cm}^{2} \mathrm{~s}^{-1}$. The $v_{\mathrm{m}}$ value averaged over the disk of the galaxy is $v_{\mathrm{m}} \approx 3 \times 10^{20} \mathrm{~cm}^{2} \mathrm{~s}^{-1}$ and

$\frac{v_{\mathrm{m}}}{v} \approx \frac{v_{\mathrm{m}}}{v_{\mathrm{turb}}} \approx 3 \times 10^{-6}$

This value is used in our calculations. Note that the calculated magnetic field strongly depends on this ratio.

\section{Calculation of the magnetic field value}

The magnetic field can be calculated using Eq. (18), in which we should set $\boldsymbol{u}=r \Omega(r) \boldsymbol{e}_{\alpha}$, where $\Omega(r)$ is the angular velocity of the differential rotation of the disk. The equations for the field components are

$\frac{\partial B_{r}}{\partial t}-v\left(\triangle B_{r}-\frac{B_{r}}{r^{2}}\right)=-\frac{4 \pi v_{\mathrm{m}}}{c} \frac{\partial j_{\alpha}^{\mathrm{cr}}}{\partial z}$,
$\frac{\partial B_{\alpha}}{\partial t}-v\left(\Delta B_{\alpha}-\frac{B_{\alpha}}{r^{2}}\right)=-\frac{4 \pi v_{\mathrm{m}}}{c}\left(\frac{\partial j_{r}^{\mathrm{cr}}}{\partial z}-\frac{\partial j_{z}^{\mathrm{cr}}}{\partial r}\right)+B_{r} r \frac{\mathrm{d} \Omega}{\mathrm{d} r}$,
$\frac{\partial B_{z}}{\partial t}-v \triangle B_{z}=-\frac{4 \pi v_{\mathrm{m}}}{c r} \frac{\partial\left(r j_{\alpha}^{\mathrm{cr}}\right)}{\partial r}$.

It follows from Eqs. (39)-(41) that the rotation does not change the components $B_{r}$ and $B_{z}$. Using the linear approximation with respect to the field and the current we can solve Eqs. (39) and (41) separately from Eq. (40). To estimate the relative contribution of the first and the second term in Eq. (40) we will solve it for two alternative cases. The first solution $B_{\alpha}=B_{\alpha}^{(1)}$ corresponds to the case $j^{\text {cr }}$ is not zero, but $\Omega(r)$ is zero. The second solution $B_{\alpha}=B_{\alpha}^{(2)}$ corresponds to the case $j^{\text {cr }}$ is zero, but $\Omega(r)$ is not zero. It will be shown in Sect. 6 that $B_{\alpha}^{(2)}$ is much larger than $B_{\alpha}^{(1)}$. At first we will solve Eqs. (39)-(41) without taking rotation into account.

We do not assume that the global large-scale magnetic field exists during the initial period of the disk's existence. However, the majority of the active stars, forming in the disk, have internal magnetic fields. We do not claim to explain the origin of the magnetic field in stars.

Magnetic fields are ejected from stars by plasma streams, such as stellar winds, and by supernova explosions. The plasma streams produce local fields in the environments of stars and create inhomogeneities in the interstellar medium. The acceleration takes place near shock fronts in the presence of the local magnetic fields. The generation of small-scale fields by accelerated particles (Völk \& McKenzie 1982; Lucek \& Bell 2000; Bell \& Lucek 2001) intensifies the acceleration process.

The acceleration time at the shock front may be estimated as

$\tau_{\mathrm{acc}} \approx \frac{\kappa}{\Delta u^{2}}$

where $\Delta u$ is the jump in velocity, and $\kappa$ is the diffusion coefficient near the front (Toptygin 1980, 1985; Berezinskii et al. 1990). Taking $\Delta u \approx 10^{8} \mathrm{~cm} \mathrm{~s}^{-1}$ and $\kappa \approx 5 \times 10^{28} \mathrm{~cm}^{2} \mathrm{~s}^{-1}$ (the last value is the average over the entire disk, see Berezinskii et al. 1990, Chapters $3 \& 6$ ) we obtain a very short time $\tau_{\text {acc }} \approx 5 \times 10^{5}$ years. In fact, $\kappa$ in a strong perturbed medium near the front is much smaller than $5 \times 10^{28} \mathrm{~cm}^{2} \mathrm{~s}^{-1}$ and $\tau_{\text {acc }}$ is shorter than $5 \times 10^{5}$ years. The obtained estimate is evidence that cosmic ray sources appear in the disk at the same moment that the supernova explosions appear. The lifetime of a type II supernova is from $10^{6}$ to $10^{7}$ years (Efremov \& Chernin 2003). The generation of the global magnetic field due to the cosmic-ray current begins just after this period. The anisotropy of the cosmic-ray diffusion is connected with the proper nonuniform structure of the interstellar medium, and with the anisotropy of the magnetic field. The magnetic-field generation connected with the anisotropic cosmic-ray diffusion becomes more effective as the field increases, and the field reaches a significant value when the cosmic rays fill the total 
volume of the disk. Estimates of the time necessary for the cosmic rays to fill the disk of the galaxy can be obtained using the well known solution for the case of a point source in a homogeneous medium

$N(r, t)=\frac{Q_{0}}{(4 \pi \kappa t)^{3 / 2}} \exp \left(-\frac{r^{2}}{4 \kappa t}\right)$

We can see from Eq. (43) that the characteristic time is $\tau \approx L^{2} / 4 \kappa$. Using the value $L \approx 10 \mathrm{kpc}$ and $\kappa \approx 5 \times 10^{28} \mathrm{~cm}^{2} \mathrm{~s}^{-1}$ we obtain $\tau \approx 5 \times 10^{8}$ years. A more realistic calculation gives the value: $3 \times 10^{6}$ years (see Berezinsky et al. 1990). The characteristic time for the field increasing can be estimated by Eq. (18):

$\frac{\partial \boldsymbol{B}}{\partial t}-v \nabla^{2} \boldsymbol{B}=\frac{4 \pi v_{\mathrm{m}}}{c} \nabla \times \boldsymbol{j}^{\mathrm{cr}}$

The term $\nabla \times[\boldsymbol{u} \times \boldsymbol{B}]$ is here omitted because it becomes important for a long time only (see Sect. 6). A solution of Eq. (44) for the case of homogeneous media can be obtained by using Green function:

$\boldsymbol{B}(\boldsymbol{r}, t)=\frac{4 \pi v_{\mathrm{m}}}{c} \int G_{B}\left(\boldsymbol{r}-\boldsymbol{r}^{\prime}, t-t^{\prime}\right) \nabla \times \boldsymbol{j}^{\mathrm{cr}}\left(\boldsymbol{r}^{\prime}, t\right) \mathrm{d}^{3} r^{\prime} \mathrm{d} t^{\prime}$

Here

$G_{B}\left(\boldsymbol{r}-\boldsymbol{r}^{\prime}, t-t^{\prime}\right)=\frac{\Theta\left(t-t^{\prime}\right)}{\left[4 \pi v\left(t-t^{\prime}\right)\right]^{3 / 2}} \exp \left\{-\frac{\left(\boldsymbol{r}-\boldsymbol{r}^{\prime}\right)^{2}}{4 v\left(t-t^{\prime}\right)}\right\}$

According to Eq. (4) the current can be expressed as a function of the particle number density:

$N(\boldsymbol{r}, t)=\int G_{N}\left(\boldsymbol{r}-\boldsymbol{r}^{\prime}, t-t^{\prime}\right) Q\left(\boldsymbol{r}^{\prime}, t^{\prime}\right) \mathrm{d}^{3} r^{\prime} \mathrm{d} t^{\prime}$

The green function $G_{N}$ has the form (46), but in this case the diffusion coefficient is a tensor $\kappa_{\mu \nu}$ with a value much larger than $v$.

$\nabla \times \boldsymbol{j}_{\alpha}^{\mathrm{cr}}=-e \kappa_{\mu \lambda} e_{\alpha \beta \mu} \frac{\partial^{2} N(\boldsymbol{r}, t)}{\partial x_{\beta} \partial x_{\lambda}}$.

The current created by the thermal component of the plasma has the same order of magnitude as the current due to cosmic rays and does not change the estimation of the time of the field increasing. Assuming that the field is zero at infinity we obtain:

$B_{\alpha}(\boldsymbol{r}, t)=-\frac{4 \pi e v_{\mathrm{m}}}{c} \kappa_{\mu \lambda} e_{\alpha \beta \mu} \frac{\partial^{2}}{\partial x_{\beta} \partial x_{\lambda}} \int \mathrm{d}^{3} r^{\prime} \mathrm{d} t^{\prime} \int \mathrm{d}^{3} r^{\prime \prime} \mathrm{d} t^{\prime \prime} G_{B}\left(\boldsymbol{r}-\boldsymbol{r}^{\prime}, t-t^{\prime}\right) G_{N}\left(\boldsymbol{r}^{\prime}-\boldsymbol{r}^{\prime \prime}, t^{\prime}-t^{\prime \prime}\right) Q\left(\boldsymbol{r}^{\prime \prime}, t^{\prime \prime}\right)$.

To estimate the $B_{\alpha}(\boldsymbol{r}, t)$ value we calculate the integral for the case of the isotropic tensor $\kappa_{\mu \lambda}=\kappa \delta_{\mu \lambda}$ :

$I(\boldsymbol{r}, t)=\int \mathrm{d}^{3} r^{\prime} G_{B}\left(\boldsymbol{r}-\boldsymbol{r}^{\prime}, t-t^{\prime}\right) G_{N}\left(\boldsymbol{r}^{\prime}-\boldsymbol{r}^{\prime}, t^{\prime}-t^{\prime \prime}\right)=\frac{1}{\pi^{3}} \int \mathrm{d}^{3} R^{\prime} \alpha^{3 / 2} \mathrm{e}^{-\alpha\left(\boldsymbol{R}-\boldsymbol{R}^{\prime}\right)^{2}-\beta R^{\prime 2}}$.

Here $\boldsymbol{R}=\boldsymbol{r}-\boldsymbol{r}^{\prime}, \boldsymbol{R}^{\prime}=\boldsymbol{r}^{\prime}-\boldsymbol{r}^{\prime \prime}$, and $\alpha=1 / 4 v\left(t-t^{\prime}\right), \beta=1 / 4 \kappa t^{\prime}$. The times $t^{\prime}$ and $t-t^{\prime}$ are of the same order of magnitude, and $v \ll \kappa$. Hence, we can take: $\alpha \gg \beta^{3 / 2}$. Performing all calculations we obtain:

$I(\boldsymbol{r}, t) \approx \frac{1}{\pi^{3 / 2}} \int_{0}^{t} \mathrm{~d} t^{\prime} \beta^{3 / 2} \mathrm{e}^{-\beta r^{2}}$.

The characteristic time $\tau$ of the field increasing is determined by $\mathrm{e}^{-\beta R^{\prime 2}}$. This gives $\tau \approx L^{2} / 4 \kappa$, where $L$ is the characteristic scale of propagation of the diffusion wave. This scale is not larger than the half-thickness of the galactic disk. In our model $L \approx 0.2$ kpc. Taking $\kappa \approx 5 \times 10^{28} \mathrm{~cm}^{2} \mathrm{~s}^{-1}$, we obtain $\tau \approx 10^{6}$ years. This is much less than the age of the galaxy. We can conclude that the field components, which are independent of the differential rotation of the disk, reach their stationary values and can be calculated using Eqs. (39)-(41), which are, in this particular case, equivalent to Eq. (19). This equation is solved in the Fourier representation:

$\boldsymbol{B}(r, z)=\frac{4 \pi v_{\mathrm{m}}}{c v} \int k^{-2}\left[\mathrm{i} \boldsymbol{k} \times \boldsymbol{j}_{\boldsymbol{k}}^{\mathrm{cr}}\right] \exp \left[\mathrm{i} k_{\perp} r \cos (\phi-\alpha)+\mathrm{i} k_{\|} z\right] \frac{k_{\perp} \mathrm{d} k_{\perp} \mathrm{d} \phi \mathrm{d} k_{\|}}{(2 \pi)^{3}}$

where the Fourier image of the current is given by Eq. (28). The quantity $\left[\mathrm{i} k \times \boldsymbol{j}_{k}^{\mathrm{cr}}\right]$ in the cylindrical coordinate system has the form

$$
\begin{aligned}
{\left[\mathrm{i} \boldsymbol{k} \times \boldsymbol{j}_{\boldsymbol{k}}^{\mathrm{cr}}\right]_{r}=} & -e Q_{\boldsymbol{k}}\left\{T_{\alpha z}\left[k_{\|}^{2}-k_{\perp}^{2} \sin ^{2}(\phi-\alpha)\right]+T_{r \alpha} k_{\|} k_{\perp} \cos (\phi-\alpha)\right\} \\
& \left.-e Q_{k}\left\{\left(T_{\alpha \alpha}-T_{z z}\right) k_{\|} k_{\perp}-T_{r z} k_{\perp}^{2} \cos (\phi-\alpha)\right\} \sin (\phi-\alpha)\right\}
\end{aligned}
$$




$$
\begin{aligned}
{\left[\mathrm{i} k \times j_{k}^{\mathrm{cr}}\right]_{\alpha}=} & e Q_{\boldsymbol{k}}\left\{T_{r z}\left[k_{\|}^{2}-k_{\perp}^{2} \cos ^{2}(\phi-\alpha)\right]+\left(T_{r r}-T_{z z}\right) k_{\|} k_{\perp} \cos (\phi-\alpha)\right\} \\
& +e Q_{k}\left\{\left[T_{r \alpha} k_{\|} k_{\perp}-T_{\alpha z} k_{\perp}^{2} \cos (\phi-\alpha)\right] \sin (\phi-\alpha)\right\},
\end{aligned}
$$

$$
\begin{aligned}
{\left[\mathrm{i} \boldsymbol{k} \times \boldsymbol{j}_{\boldsymbol{k}}^{\mathrm{cr}}\right]_{z}=} & e Q_{\boldsymbol{k}} k_{\perp}^{2}\left\{T_{r \alpha}\left[2 \cos ^{2}(\phi-\alpha)-1\right]+\left(T_{\alpha \alpha}-T_{r r}\right) \sin (\phi-\alpha) \cos (\phi-\alpha)\right\} \\
& +e Q_{k} k_{\|} k_{\perp}\left\{T_{\alpha z} \cos (\phi-\alpha)-T_{r z} \sin (\phi-\alpha)\right\}
\end{aligned}
$$

Here

$T_{\mu v}=\frac{\kappa_{\mu v}}{\widetilde{\kappa}}-\frac{D_{\mu v}^{\mathrm{p}}}{\widetilde{D}_{\mathrm{p}}}$

and

$\widetilde{\kappa}=\left[\kappa_{\alpha \alpha}+\left(\kappa_{r r}-\kappa_{\alpha \alpha}\right) \cos ^{2}(\phi-\alpha)+2 \kappa_{r \alpha} \cos (\phi-\alpha) \sin (\phi-\alpha)\right] k_{\perp}^{2}$

$$
+2\left[\kappa_{r z} \cos (\phi-\alpha)+\kappa_{\alpha z} \sin (\phi-\alpha)\right] k_{\perp} k_{\|}+\kappa_{z z} k_{\|}^{2} \text {. }
$$

The analogous expression is true for $\widetilde{D}_{\mathrm{p}}$.

The integration of Eqs. (39)-(41) is very complicated, but we can use reasonable approximations, such as the fact that expression (55) is positive for all $\phi$ values, which becomes obvious in the coordinate system where the tensor $\kappa_{\mu \nu}$ is diagonal. In this case $\widetilde{\kappa}=\kappa_{\perp} k_{\perp}^{\prime 2}+\kappa_{\|} k_{\|}^{\prime 2}>0$. The relative change of $\widetilde{\kappa}$ is small in the interval $0 \leq \phi \leq 2 \pi$. This makes it possible to use the $\widetilde{\kappa}$ value averaged over the angle $\phi$, i.e.

$\overline{\widetilde{\kappa}}=\frac{1}{2}\left(\kappa_{\alpha \alpha}+\kappa_{r r}\right) k_{\perp}^{2}+\kappa_{z z} k_{\|}^{2} \equiv \kappa_{z z}\left(k_{\|}^{2}+a^{2} k_{\perp}^{2}\right), \quad a=\sqrt{\frac{\kappa_{\alpha \alpha}+\kappa_{r r}}{2 \kappa_{z z}}}$.

A similar change has to be made in both denominators in Eq. (54)

$\overline{\widetilde{D}_{\mathrm{p}}}=D_{z z}^{\mathrm{p}}\left(k_{\|}^{2}+b^{2} k_{\perp}^{2}\right), \quad b=\sqrt{\frac{D_{\alpha \alpha}^{\mathrm{p}}+D_{r r}^{\mathrm{p}}}{2 D_{z z}^{\mathrm{p}}}}$

The integrals containing $\sin (\phi-\alpha)$ are zero. The integral over the angle $\phi$ was taken using the formula $\int_{0}^{2 \pi} \exp \left(\mathrm{i} k_{\perp} r \cos \varphi\right) \mathrm{d} \varphi=$ $2 \pi J_{0}\left(k_{\perp} r\right)$. The integrals over $k_{\|}$can be calculated in the complex plane inside the contour of the infinite radius. The integral over $k_{\perp}$ from 0 to $\infty$ was taken by the formula $\int_{0}^{\infty} \exp \left(-k_{\perp} R\right) J_{0}\left(k_{\perp} r\right) \mathrm{d} k_{\perp}=\sqrt{r^{2}+R^{2}}$. Here $J_{0}\left(k_{\perp} r\right)$ is a Bessel function. After performing all integrations we obtain the expressions for the field values using the small parameter $(|z| \ll R)$

$$
\begin{aligned}
B_{r}(r, z)= & B_{0}(r)\left\{\left(\frac{\kappa_{\alpha z}}{\kappa_{z z} a(a+1)}-\frac{D_{\alpha z}^{\mathrm{p}}}{D_{z z}^{\mathrm{p}} b(b+1)}\right)\left(1-\frac{R}{\sqrt{r^{2}+R^{2}}}\right) \frac{R}{r}+\left(\frac{\kappa_{\alpha z}(a-1)}{\kappa_{z z} a(a+1)}-\frac{D_{\alpha z}^{\mathrm{p}}(b-1)}{D_{z z}^{\mathrm{p}} b(b+1)}\right) \frac{r}{\sqrt{r^{2}+R^{2}}}\right. \\
& \left.-\left(\frac{\kappa_{\alpha z}}{\kappa_{z z}(a+1)}-\frac{D_{\alpha z}^{\mathrm{p}}}{D_{z z}^{\mathrm{p}}(b+1)}\right) \frac{r R f(z)}{\left(r^{2}+R^{2}\right)^{3 / 2}}+\left(\frac{\kappa_{r \alpha}}{\kappa_{z z}(a+1)}-\frac{D_{r \alpha}^{\mathrm{p}}}{D_{z z}^{\mathrm{p}}(b+1)}\right) \frac{r^{2} z}{\left(r^{2}+R^{2}\right)^{3 / 2}}\right\},
\end{aligned}
$$$$
B_{\alpha}^{(1)}(r, z)=B_{0}(r)\left\{\left(\frac{\kappa_{r z}}{\kappa_{z z} a(a+1)}-\frac{D_{r z}^{\mathrm{p}}}{D_{z z}^{\mathrm{p}} b(b+1)}\right)\left(1-\frac{R}{\sqrt{r^{2}+R^{2}}}\right) \frac{R}{r}+\left(\frac{\kappa_{r z}}{\kappa_{z z}(a+1)}-\frac{D_{r z}^{\mathrm{p}}}{D_{z z}^{\mathrm{p}}(b+1)}\right) \frac{r}{\sqrt{r^{2}+R^{2}}}\right.
$$$$
\left.-\left(\frac{\kappa_{r z}}{\kappa_{z z}}-\frac{D_{r z}^{\mathrm{p}}}{D_{z z}^{\mathrm{p}}}\right) \frac{r R f(z)}{\left(r^{2}+R^{2}\right)^{3 / 2}}-\left(\frac{\kappa_{r r}-\kappa_{z z}}{\kappa_{z z}(a+1)}-\frac{D_{r r}^{\mathrm{e}}-D_{z z}^{\mathrm{p}}}{D_{z z}^{\mathrm{p}}(b+1)}\right) \frac{r^{2} z}{\left(r^{2}+R^{2}\right)^{3 / 2}}\right\} \text {, }
$$

$$
\begin{aligned}
B_{z}(r, z)= & B_{0}(r)\left\{\left(\frac{\kappa_{r \alpha}}{\kappa_{z z} a(a+1)}-\frac{D_{r \alpha}^{\mathrm{p}}}{D_{z z}^{\mathrm{p}} b(b+1)}\right)\left[\left(1-\frac{R}{\sqrt{r^{2}+R^{2}}}\right) \frac{2 R}{r}-\frac{r}{\sqrt{r^{2}+R^{2}}}\right]\right. \\
& \left.-\left(\frac{\kappa_{\alpha z}}{\kappa_{z z}(a+1)}-\frac{D_{\alpha z}^{\mathrm{p}}}{D_{z z}^{\mathrm{p}}(b+1)}\right) \frac{r^{2} z}{\left(r^{2}+R^{2}\right)^{3 / 2}}\right\} .
\end{aligned}
$$

Here $B_{0}(r)$ and $f(z)$ are dimensional factors,

$$
B_{0}(r)=\frac{e Q_{0} v_{\mathrm{m}}}{c v_{\mathrm{turb}} r}, \quad f(z)=\left\{\begin{array}{l}
|z|, \quad|z|>h, \\
h\left[1-\frac{2}{\pi} \cos \left(\frac{\pi z}{2 h}\right)\right], \quad|z|<h .
\end{array}\right.
$$


The values (58)-(60) of the magnetic field can be reached in a time necessary for the cosmic rays to fill the galactic volume, i.e., in a time less than $10^{8}$ years.

Consider now the field value in the vicinity of the solar system $\left(r=10 \mathrm{kpc}, R=16 \mathrm{kpc}, z \approx h=0.2 \mathrm{kpc}, Q_{0}=10^{43}\right.$ particle $/ \mathrm{s}$, the ratio of magnetic viscosities is given by Eq. (38)).

Under the assumptions above, the dimensional factor $B_{0}(r)$ has the value

$B_{0}(r) \approx 1.5 \times 10^{-5} G$.

The dimensionless factors in expressions (58)-(60), which depend on $r$ and $z$, can be divided into two groups. The terms that are independent of $z$ are of the order of one, while the terms that are dependent on $z$ are of the order of $h / R \approx 10^{-2}$. All terms have factors such as

$$
\frac{\kappa_{\alpha z}}{\kappa_{z z} a(a+1)}-\frac{D_{\alpha z}^{\mathrm{p}}}{D_{z z}^{\mathrm{p}} b(b+1)} \text {. }
$$

This expression depends on the ratios of the components of the diffusion tensors. This expression is not zero if the off-diagonal components of the diffusion tensors are not zero. The general definition of the diffusion coefficient is $D_{\mu \nu}=\left\langle L_{\mu} L_{\nu}\right\rangle / \tau$, where $L_{\mu}$ is the path of the particle in a time $\tau$. If the propagation in the $\mu$ direction is independent of the propagation in the $v$ direction then $\left\langle L_{\mu} L_{\gamma}\right\rangle=0$. The nonuniform structure of the galactic medium and the complicated magnetic field structures make $\left\langle L_{\mu} L_{\gamma}\right\rangle \neq 0$ (see tensor (37)). The diffusion coefficients for relativistic particles are not correlated with those for thermal particles. Thus the two terms in (63) cannot cancel each other out. Using the estimate of $\kappa_{\|}$and $\kappa_{\perp}$, which are of the same order of magnitude, and taking $\cos \theta \approx \cos \gamma \approx 0.1$, we obtain

$\frac{\kappa_{\alpha z}}{\kappa_{z z} a(a+1)}-\frac{D_{\alpha z}^{\mathrm{p}}}{D_{z z}^{\mathrm{p}} b(b+1)} \geq 10^{-2}$.

Using this estimate for expressions (51)-(55), we obtain the following values for the poloidal field components:

$B_{r} \approx B_{z} \approx 1.5 \times 10^{-7} \mathrm{G}$.

The obtained values of $B_{r}$ and $B_{z}$ are about one tenth of the observed large-scale toroidal field value in the disk. The same estimate is true for that part of the toroidal component $B_{\alpha}^{(1)}$ which is independent of rotation and is described by formula (59). The poloidal field without the toroidal field is unstable. The total toroidal field is calculated in the next section.

\section{The differential rotation and the toroidal field}

The differential rotation of the disk stretches the $B_{r}$ component of the poloidal field in the direction of the linear velocity of the medium and transforms it into the toroidal field $B_{\alpha}^{(2)}$. This component can be calculated using Eq. (40) where we have to put $j^{\text {cr }}=0$ :

$\frac{\partial B_{\alpha}^{(2)}}{\partial t}=v\left(\Delta B_{\alpha}^{(2)}-\frac{B_{\alpha}^{(2)}}{r^{2}}\right)+B_{r}(r, z) \frac{\mathrm{d} \Omega}{\mathrm{d} r} r$.

The component $B_{r}$ is determined by expressions (58) and (64) and is considered here as a known quantity.

Our galaxy is relatively young (about $10^{10}$ years old) and there were not too many revolutions of the disk. This makes it possible to solve Eq. (65) by the method of subsequent approximations. In the first approximation we can disregard the dissipation, keeping in mind that the dissipation has no time to evolve. The remaining equation has the solution

$B_{\alpha}^{(2)}(r, z, t)=B_{r}(r, z) \frac{\mathrm{d} \Omega}{\mathrm{d} r} r t$.

This approximation is applicable if the omitted dissipative term is not larger than the remaining term. Substituting the solution (66) in Eq. (65) we can see that the term containing $f(z)$ in Eq. (58) provides the main contribution to the omitted part of equation. The omitted part is of the order of

$\frac{v t}{h r} B_{r} \frac{\mathrm{d} \Omega}{\mathrm{d} r} r$

That means that the solution (66) is applicable for the time $t<h r / v$. Using the numerical values we obtain $t<6 \times 10^{16} \mathrm{c} \approx 6 \times 10^{9}$ years. This time interval is apparently close to the age of the galactic disk. That means that the solution (66) can approximately describe the toroidal field in the recent time. Using the value

$\frac{\mathrm{d} \Omega}{\mathrm{d} r} r \approx 30 \mathrm{~km} \mathrm{~s}^{-1} \times \mathrm{kpc} \approx 10^{-15} \mathrm{~s}^{-1}$ 
for the distance $r=10 \mathrm{kpc}$ (see Ruzmaikin et al. 1988), taking $t \approx 6 \times 10^{9}$ years, and $B_{r}=1.5 \times 10^{-7} \mathrm{G}$ we obtain $B_{\alpha}=9 \times 10^{-6} \mathrm{G}$. So the rotation causes a significant increase of the toroidal field. Taking into account the approximations used in the calculations and the uncertainty of the observations we can say that the obtained field value corresponds to that observed: $B_{\text {obs }} \approx(2-3) \times 10^{-6}$ $\mathrm{G}$ (see, for example, Beck 2001). The geometry of the magnetic field and the field values are approximately in agreement with the estimates of the diffusion coefficients accepted in Sect. 4. The poloidal components are an order of magnitude smaller than the toroidal ones. Both the poloidal and toroidal components are necessary to provide the anisotropy of the diffusion coefficients.

The consideration of the energy balance is additional evidence in favor of the proposed model of field generation. The time required for the magnetic field and turbulence regeneration by the energy input from supernova explosions is about $10^{7}$ years. This time is enough for the supernova remnants to fill the volume of the disk (one explosion per 30 years or a little less frequently) and for the magnetic field to become turbulent. This time is also enough to create cosmic rays with a total energy on the order of $3 \times 10^{40} \mathrm{erg} \mathrm{s}^{-1} \times 10^{14} \mathrm{~s} \approx 3 \times 10^{54} \mathrm{erg}$. The energy required to restore the regular magnetic field is $B^{2} V_{\mathrm{d}} / 8 \pi \approx 1.6 \times 10^{54} \mathrm{erg}$, if the disk volume is $V_{\mathrm{d}} \approx 4 \times 10^{66} \mathrm{~cm}^{3}$. This means that the energy of cosmic rays is sufficient to support the observed magnitude of the galactic large-scale magnetic field.

The considered process of toroidal field amplification does not lead to an additional dissipation of the field. Equation (65) has a stationary solution for $t \rightarrow \infty$. This solution can be presented in the form

$B_{\alpha}^{(2)}(r, z)=\int_{-\infty}^{\infty} \bar{B}_{\alpha}(r, \lambda) \exp (\mathrm{i} \lambda z) \frac{\mathrm{d} \lambda}{2 \pi}$

The Fourier transform of expression (67) with respect to the variable $z$ is calculated using the stationary variant of Eq. (65):

$\bar{B}_{\alpha}^{(2)}(r, \lambda)=I_{1}(|\lambda| r) \int_{r}^{\infty} K_{1}\left(|\lambda| r^{\prime}\right) \bar{B}_{r}\left(r^{\prime}, \lambda\right) \frac{r^{\prime 2}}{v} \frac{\mathrm{d} \Omega}{\mathrm{d} r^{\prime}} \mathrm{d} r^{\prime}+K_{1}(|\lambda| r) \int_{0}^{r} I_{1}(|\lambda| r) \bar{B}_{r}\left(r^{\prime}, \lambda\right) \frac{r^{\prime 2}}{v} \frac{\mathrm{d} \Omega}{\mathrm{d} r^{\prime}} \mathrm{d} r^{\prime}$.

Here $I_{1}(x), K_{1}(x)$ are Bessel functions, and $\bar{B}_{r}(r, \lambda)$ is the Fourier image of the $r$ component of the field. Expression $(64)$ can be used for the field estimate which gives a stationary solution

$\left.B_{\alpha}^{(2)}\right|_{t \rightarrow \infty} \approx B_{r} \frac{r^{2}}{v} \frac{\mathrm{d} \Omega}{\mathrm{d} r} r \approx 1.5 \times 10^{-3} \mathrm{G}$

This large value of the field could be reached in the far future $t \gg h r / v \approx 6 \times 10^{9}$ years, however, in this case the energy density of the field becomes larger than the energy density of the interstellar plasma, and nonlinear effects become important.

\section{Conclusions}

The proposed model for the large-scale galactic magnetic field generation is a good example of self-organization in a strong non-equilibrium interstellar plasma. Discrete sources in the disk, such as exploding stars, produce relativistic particles which perturb the ambient plasma and create the electric current. The nonsymmetric electric current generates a magnetic field with a space scale much larger than the scale of regions occupied by the sources of the current. The large-scale field changes the kinetic coefficients of the ambient plasma, introducing anisotropy into the system and supporting, in this way, the anisotropy which is necessary for the field generation. The anisotropy of the distribution of the electric current is connected not only with the smallscale magnetic field structure but also with the geometry of the galaxy that leads to maximum leaking of the relativistic particles perpendicular to the plane of the disk. The process considered does not need an initial large-scale "seed" field to start the field generation. The existence of small-scale magnetic fields ejected from stars is sufficient to start the process of particle acceleration up to relativistic energies. Then, the cosmic ray propagation in the galaxy creates an electric current and corresponding large-scale magnetic field.

The simple model considered in this paper shows that the processes of generation and propagation of relativistic particles (cosmic rays), together with the differential rotation of the disk, are sufficient to explain the structure and value of the observed large-scale magnetic field in the galaxy avoiding the $(\alpha \Omega)$ dynamo process. The proposed mechanism can generate a magnetic field also in galaxies where exploded stars are present but differential rotation and turbulence helicity are insignificant.

The next step in the development of this theory should include a more realistic model of the galaxy, a more precise definition of diffusion coefficients for the magnetic field and particles and finally, the solution of the self-consistent problem. The magnetic field generated by cosmic rays, gyrotropic turbulence, and differential rotation should be calculated together with the coefficients of diffusion of the field and of the particles.

Acknowledgements. The authors are thankful to B. V. Kuteev, V. A. Rozhansky and L. D. Tsendin for the discussion of the plasma aspects of the theory. The paper was performed with the partial support of the Russian Foundation of Basic Research (grant 01-02-16654). 


\section{References}

Alfven, H., \& Fälthammar, C.-G. 1963, Cosmical Electrodynamics (Oxford: Claredon Press)

Beck, R., \& Brandenburg, A., Moss, D., et al. 1996, ARA\&A, 34, 155

Beck, R. 2001, Space Sci. Rev., 99, 243

Berezinsky, V. S., Bulanov, S. V., Dogiel, V. A., et al. 1990, Astrophysics of Cosmic Rays, ed. V. L. Ginzburg (Amsterdam: North-Holland) Bykov, A. M., \& Toptygin, I. N. 1992, Sov. Phys. JETP, 74, 3

Elsasser, W. M. 1946, Phys. Rev., 69, 106

Grusinov, A., \& Diamond, P. 1994, Phys. Rev. Lett., 72, 1651

Grusinov, A., \& Diamond, P. 1996, Phys. Plasm., 3, 1853

Kulsrud, R. M., \& Anderson, S. W. 1992, ApJ, 396, 606

Parker, E. N. 1979, Cosmical Magnetic Fields (Oxford: Claredon Press)

Rusmaikin, A. A., Sokoloff, D. D., \& Shukurov, A. M. 1988, Galactic Magnetic Fields (Moscow: Nauka)

Vainstein, S. I., Zeldovich, Y. B., \& Rusmaikin, A. A. 1980, Turbulent Dynamo in Astrophysics (Moscow: Nauka)

Vainstein, S. I., \& Rossener, R. 1991, ApJ, 376, 199

Vainstein, S. I., \& Cattaneo, F. 1992, ApJ, 388, 17

Völk, H. J., \& McKenzie, J. M. 1982, A\&A, 116, 191

Zeldovich, Ya. B. 1956, JETP, 31, 154 